\title{
The Antioxidant Butylated Hydroxytoluene Prevents Early Cholesterol-induced Microcirculatory Changes in Rabbits
}

\author{
R. J. Xiu, * A. Freyschuss, ${ }^{\star}$ X. Ying, ${ }^{\star}$ L. Berglund, ${ }^{\star}$ P. Henriksson, ${ }^{\star}$ and I. Björkhem \\ ${ }^{*}$ Clinical Research Center and Departments of ${ }^{\ddagger}$ Medicine and ${ }^{\S}$ Clinical Chemistry, Karolinska Institutet, \\ Huddinge Hospital, 14186 Huddinge, Sweden
}

\begin{abstract}
Microcirculation was studied during 10 wk in untreated rabbits $(n=13)$ and in rabbits treated with dietary addition of $1 \%$ cholesterol $(n=13), 1 \%$ cholesterol $+1 \%$ of the antioxidant BHT (butylated hydroxytoluene) $(n=11)$, or 1\% BHT ( $n$ $=5$ ). The studies were performed by direct intravital microscopic imaging of the left and right conjunctivae with the use of a stereo microscope and a high resolution television camera. Microvessel diameter, erythrocyte flow velocity, and microhemorheologic conditions were evaluated quantitatively via a computer-assisted digital image processing system.

Significant and marked changes occurred in all the above variables as a consequence of cholesterol feeding. After 3 wk of feeding there was a dramatic decrease $(\sim 30 \%)$ in blood flow velocity in arterioli of the third order $(P<0.0001)$, accompanied by aggregation of cells in $40-50 \%$ of the smaller conjunctival vessels $(P<0.0001)$. These changes were enhanced further during the following $7 \mathrm{wk}$ of treatment. All the above changes in the microcirculation were markedly reduced by the addition of BHT treatment. The diameter of the above arterioli decreased in the purely cholesterol-fed group $(P<0.005)$, whereas this did not occur in the group fed both cholesterol and BHT. In rabbits fed BHT in the absence of cholesterol, there was no significant effect on any assessed microcirculatory variable.

In conclusion, the results demonstrate that the antioxidant BHT prevented early cholesterol-induced microcirculatory changes. This prevention occurred in the absence of a reduction of blood lipid levels. The results provide strong support for the hypothesis that a considerable part of the effects on microcirculation in hypercholesterolemia may be due to cholesterol-induced oxidations and not to cholesterol itself. The results are discussed in relation to the previously demonstrated antiatherogenic effect of BHT and the possible use of antioxidants in the therapy and prophylaxis of atherosclerosis. (J. Clin. Invest. 1994. 93:2732-2737.) Key words: microcirculation • hypercholesterolemia $\bullet$ atherosclerosis $\bullet$ blood flow velocity $\bullet$ arteriolar diameter
\end{abstract}

Address correspondence to Dr. Ingemar Björkhem, Department of Clinical Chemistry, Karolinska Institutet, Huddinge Hospital, 14186 Huddinge, Sweden.

Received for publication 4 December 1992 and in revised form 28 February 1994

J. Clin. Invest.

(C) The American Society for Clinical Investigation, Inc.

0021-9738/94/06/2732/06 \$2.00

Volume 93, June 1994, 2732-2737

\section{Introduction}

The most obvious result of atherosclerosis is noticed in the large arteries, the atherosclerotic plaques. Much is known concerning the sequence of their development, and every month new information is unraveled about the intricate interaction between cells and cytokines in the vessel wall. The microcirculatory system of arterioles, capillaries, and venules has attracted much less interest. The limited interest in microcirculation is surprising in view of the fact that the general consequences of a compromised flow in the arteries can be expected to be reflected in this system. It is possible that early microcirculatory changes may affect the susceptibility for degenerative changes in the arteries. Changes in the microcirculatory system due to the atherosclerotic process might even precede the changes known to occur in the arterial system. Thus, dietaryinduced hypercholesterolemia in rats has been shown recently to result in microvascular (and large artery) dysfunction before development of histological signs associated with atherosclerosis (1).

There are relatively few published studies on cholesterol-induced changes in the microcirculation (1-4), and the mechanism behind these changes has not been completely clarified. However, it has been reported that hypercholesterolemia modifies the macromolecular leakage from postcapillary venules in response to serotonin (1). In rats, hypercholesterolemia may cause an early depression of the response to endothelium-derived relaxing factor (EDRF) ${ }^{1}$ in microvessels (1). Evidence has also been presented that hypercholesterolemia may impair endothelium-dependent dilation of the coronary microcirculation in patients and that this impairment can be restored by short-term administration of arginine (4).

In this study, we demonstrate early and dramatic cholesterol-induced changes in the microcirculation of rabbits. A substantial part of these changes could be prevented by addition of the antioxidant butylated hydroxytoluene (BHT) to the diet. We have shown previously that this treatment prevents the development of atherosclerosis in the same animal model (5). Thus, oxidative mechanisms might be important not only for modification of lipoproteins (6) but also for the microcirculation.

\section{Methods}

Animals, feeding, and observation conditions. 44 young male New Zealand White rabbits, with an average weight of $2.6 \mathrm{~kg}$, were housed individually under conditions of $12-\mathrm{h}$ light/dark periods. 13 rabbits

1. Abbreviations used in this paper: BHT, butylated hydroxytoluene; EDRF, endothelium-derived relaxing factor. 
were fed standard chow during 10 wk. Another 13 rabbits were fed standard chow supplemented with $1 \%(\mathrm{wt} / \mathrm{wt}$ ) cholesterol. 13 rabbits were fed a diet supplemented with both $1 \%$ cholesterol and $1 \%$ (wt $/ \mathrm{wt}$ ) BHT only. Cholesterol and BHT were pelleted into the rabbit chow without using solubilizing vehicles.

Initially, as well as after 3,6, and $10 \mathrm{wk}$, the microcirculatory vessels of the conjunctival plexus of both eyes of each rabbit were examined by a long focus stereo microscope. The images were recorded with a video camera (compare below). Efforts were made to put the focus to the same area of conjunctiva on each eye of all the rabbits. A total observation time of a minimum of 2 min was used for each rabbit eye. The observations were made without actually touching the conjunctiva, under awake conditions, and without anesthesia or other drugs. The animals were placed in a standard box for blood sampling during the observations. The recordings and evaluations were performed without any knowledge to which feeding group each rabbit belonged, and rabbits from the four groups were mixed in random order.

One of the rabbits in the group fed cholesterol and BHT had to be excluded from the study because of a spinal injury. Another rabbit from this group was excluded because of an unclear microscopic view from the start of the study and onwards.

Distribution of microvessels on the bulbar conjunctiva. The main artery in the conjunctiva, the anterior ciliaric artery, penetrates the sclera and enters the bulb in front of the limbus. Before penetration, the anterior conjunctival artery branches off. This study was made on arterioli of the third order belonging to the anterior conjunctival artery. In addition, the true capillary network at the limbus of the cornea was studied.

Equipment and methods used for image processing. The microcirculatory events in the conjunctivae of the rabbits were recorded using a video camera (CCD AXC-D7CE; Sony) and a videocassette recorder (SLV-373; Sony) and were viewed on a high-resolution color monitor (PVM-1443 MD; Sony). The system is based on an IBM computer $(386 / 40)$. A dynamic and still imaging separation processing mode was designed for development of a digital microcirculation image workstation. A special video digitizer (15 $\mathrm{MHz}$ alternating/direct current, $4 \times 512 \times 512 \times 8$ bits framebuffer and RGB digital analogue outputs) was used for on-line grabbing of the video microscopy images as well as for on-line dynamic image processing. The system and its use have been described recently in detail elsewhere (7). The system allows the microvascular contour line and diameter, the blood flow velocity, and other variables to be extracted from any area of the network and measured. Furthermore, the procedure also includes a special images shading function for correcting erasing vestiges of the assembly and heavy complicated background.

Measurement of microvessel diameter. The microvessel diameter (arterioli of the third order belonging to the anterior conjunctival artery) was measured with the above equipment using an autotracking measuring method (8). For a more detailed description of the general methodology see reference 9 . Three independent measurements were made in each eye on each occasion. The arteriolar diameter $(\mu \mathrm{m})$ was thus calculated as a mean of six measurements for each rabbit on each occasion. The reproducibility and intraindividual variation were tested by duplicate blinded measurements on 13 individual rabbits on two occasions. The coefficient of variation was found to be $8.4 \%$.

Measurement of blood cell flow velocity. Using the above equipment, a computer-generated flying spot technique was used to determine blood cell flow velocity in the microvessel (arterioli of the third order belonging to the anterior conjunctival artery) $(7,10,11)$. In this method, a flying spot is positioned close to the capillary of interest by means of horizontal and vertical position controls. The speed of the flying spot is adjusted until corresponding to the velocity of the optical signals in the capillary. Before the measurements, the total magnification coefficient of the microscope video imaging system was obtained by using a micrometer to define the pixel length $(\mu \mathrm{m} / \mathrm{pixel})$. The velocity of the flying spots was calibrated by a standard velocity generator (model 210; IPM, Inc., San Diego, CA). Based on the calibration, the accuracy of this method was found to be $0.07 \mathrm{~mm} / \mathrm{s}$ at velocities ranging from 0 to $1 \mathrm{~mm} / \mathrm{s}$. Also in this case, three independent measure- ments were made in each eye on each occasion. The blood flow velocity was thus calculated as a mean of six measurements for each rabbit at each occasion. The reproducibility and intraindividual variation were tested by duplicate blinded measurements on 13 rabbits on two occasions. The coefficient of variation was found to be $8.8 \%$.

Evaluation of stasis, aggregation, and segmental dilation. All the evaluations were made with the above equipment. Efforts were made to put the focus to the same area of conjunctiva on each eye of all the rabbits. The area investigated was always $9 \mathrm{~mm}^{2}$ in each eye and always included the arterioli of the third order belonging to the anterior conjunctival artery. Observations were always made on both eyes in each individual rabbit.

Stasis was defined as a condition in which the cellular elements were retained as a mass of blood cells interrupting flow (12). Stasis was classified into the following four groups: (1) no stasis; (2) presence of portions of stased blood flow in vessels with diameter $<20 \mu \mathrm{m}$ or a flow velocity of $<300 \mu \mathrm{m} / \mathrm{s}$ in the capillaries; (3) presence of continuously stased blood flow in vessels with a diameter $<20 \mu \mathrm{m}$ or extremely slow flow velocity in the capillaries; and (4) presence of continuously stased blood in the whole corneal plexus network (vessels beyond arterioli of the third degree).

Aggregation was defined as the presence of clumps of cells that are sufficiently cohesive to circulate as a solid mass (12). Aggregation was classified into the following four groups: (1) no aggregation; (2) occasional presence of aggregates in the small microvessels with a diameter $<20 \mu \mathrm{m}$; (3) aggregates in up to $50 \%$ of the microvessels with a diameter $<30 \mu \mathrm{m}$; and (4) aggregation in $>50 \%$ of the microvessels with a diameter $<30 \mu \mathrm{m}$.

Segmental dilation was identified as irregularly dilated segments pre- or postcapillary with a length varying between 50 and $200 \mu \mathrm{m}$. More than one segmental dilation in each microvessel was never observed. This variable was classified as follows: $(1)$ no segmental dilation; (2) segmental dilation in less than five vessels in the two areas (2 $\times 9 \mathrm{~mm}^{2}$ ) corresponding to the two eyes; and (3) segmental dilation in five or more of the vessels observed.

Measurement of cholesterol and lipid levels. Blood samples were drawn from an ear artery of each rabbit at $0,3,6$, and 10 wk. After centrifugation, the plasma samples were analyzed for cholesterol and triglycerides using standard enzymatic procedures. The hyperlipidemic sera were diluted before the assay, and under the conditions used there was a linear relation between photometric response and amount of serum. Commercially available enzymatic assays (Boehringer Mannheim GmbH, Mannheim, Germany) were used for the quantitations.

Ethics. The project was approved by the Animal Ethical Committee in Stockholm.

Statistical methods. All statistical analyses were performed by ANOVA. Parametric or nonparametric ANOVA was used when appropriate. The first analysis was always an overall ANOVA taking all the groups and measurements into account. If this analysis showed that the groups differed, between group differences were analyzed. Values are means \pm SEM unless otherwise stated.

\section{Results}

Lipid levels. During the experiment, the weight of the four sets of rabbits increased from an average of $2.6 \pm 0.1$ to $3.4 \pm 0.1 \mathrm{~kg}$. There was no significant difference between the groups. Plasma lipid levels increased in the two sets of rabbits given cholesteroland cholesterol/BHT-enriched pellets, whereas no increase in either cholesterol or triglycerides was seen in the plasma of the rabbits fed standard rabbit chow or pellets enriched with BHT only. The rabbits fed cholesterol-supplemented pellets increased their cholesterol values from $1.5 \pm 0.1$ to $80.3 \pm 7.1$ $\mathrm{mmol} /$ liter (Table I) and triglycerides from $1.4 \pm 0.1$ to $3.6 \pm 0.8$ $\mathrm{mmol} /$ liter. The rabbits fed with a supplement of both cholesterol and BHT increased from $1.4 \pm 0.2$ to $85.4 \pm 5.7 \mathrm{mmol} /$ liter in cholesterol (Table I) and from $1.3 \pm 0.1$ to $5.7 \pm 1.4 \mathrm{mmol} /$ 
Table I. Effect of Dietary Cholesterol (1\%), BHT (1\%), and Cholesterol (1\%)/BHT (1\%) on Circulating Levels of Cholesterol in the Rabbits

\begin{tabular}{ccccc}
\hline & \multicolumn{5}{c}{$\begin{array}{c}\text { Cholesterol levels after different } \\
\text { duration of treatment }\end{array}$} \\
\cline { 2 - 5 } Group & $0 \mathrm{wk}$ & $3 \mathrm{wk}$ & $6 \mathrm{wk}$ & $10 \mathrm{wk}$ \\
\hline & \multicolumn{5}{c}{ mmol/liter } \\
Controls $(n=13)$ & $1.1 \pm 0.1$ & $0.8 \pm 0.1$ & $0.8 \pm 0.2$ & $0.6 \pm 0.1$ \\
$\begin{array}{c}\text { BHT-treated }(n=5) \\
\text { Cholesterol-treated } \\
(n=13)\end{array}$ & $1.3 \pm 0.1$ & $1.1 \pm 0.1$ & $1.0 \pm 0.1$ & $0.9 \pm 0.2$ \\
$\begin{array}{c}\text { Cholesterol/BHT- } \\
\text { treated }(n=11)\end{array}$ & $1.5 \pm 0.1$ & $36.3 \pm 4.9$ & $63.1 \pm 4.4$ & $80.3 \pm 7.1$ \\
& $1.4 \pm 0.2$ & $38.8 \pm 5.8$ & $63.5 \pm 6.1$ & $82.7 \pm 5.4$ \\
\hline
\end{tabular}

Values are means \pm SEM.

liter in triglycerides. The cholesterol and triglyceride levels were not significantly different between the latter two groups of rabbits $(P>0.05)$.

Microcirculatory changes. During the course of the study, several changes in the microcirculation were observed. An early finding was a decrease in blood flow velocity $(P<0.0001)$ among the cholesterol-fed rabbits that persisted throughout the study (Fig. 1). Similar changes, although not to the same extent, were later observed also in the BHT/cholesterol-treated rabbits. However, there were significant differences both in the level $(P<0.0001)$ and overall pattern change $(P<0.0001)$ between the latter two rabbit groups. In contrast, no changes in blood flow velocity were found in the control animals or in the animals fed BHT only.

The cholesterol-fed animals also showed an increased stasis in the vessels compared with the BHT/cholesterol-fed animals $(P=0.0003)$ (Fig. 2). Again, this was seen only rarely in the control group and in the rabbits treated with BHT only. Another early change, most pronounced among the cholesterolfed rabbits, was an increased aggregation of erythrocytes in the microvessels with diameters $<30 \mu \mathrm{m}(P<0.0001)$. As seen in Fig. 3, there was a significant difference between the animals fed BHT/cholesterol and those fed cholesterol only ( $P$ $<0.0001)$. Aggregation was seen only rarely in the control rabbits and in the rabbits treated with BHT only.
The arteriolar diameter in the control animals showed a slight increase during the $10 \mathrm{wk}$ of observation (Fig. 4). The same tendency was also seen in the rabbits treated with BHT only and cholesterol/BHT $(P<0.05)$. In contrast, rabbits treated with cholesterol only had a clear decrease in arteriolar diameter when compared with both the control group and the initial level $(P<0.005)$. The arteriolar diameter in the animals fed BHT only was not different from that of the control animals $(P>0.05)$. No significant changes were seen in the average diameter of the venous vessels in any of the groups (results not shown).

There was an increased number of segmental dilations of the microvessels in the cholesterol-fed rabbits as compared with the controls $(P<0.0001)$ (Fig. 5). Similar findings were seen among the BHT/cholesterol-treated rabbits, but there was a tendency for fewer changes. However, the difference between the latter two groups did not reach statistical significance $(P$ $>0.05$ ). The capillary network became successively less dense with time among the cholesterol-fed animals, indicating a reduction in the number of visible capillaries. Less marked changes were observed in the rabbits fed cholesterol/BHT and were not seen at all in the control animals or in the animals treated with BHT only (latter results not shown).

\section{Discussion}

In this investigation we were able to study early microcirculatory changes through intravital microscopy of conjunctival vessels in rabbits with a dietary-induced hyperlipidemia and to compare them with the microcirculation in rabbits on standard rabbit chow. Furthermore, we studied effects of the antioxidant BHT added to the same hypercholesterolemic diet as above.

Among the different microcirculatory parameters studied, blood flow velocity and arteriolar diameter are most accurately defined from a quantitative point of view. Degree of aggregation, occurrence of stasis, segmental dilation, and density of the capillary network are more difficult to evaluate in quantitative terms. It should be emphasized that all the different measurements and evaluations were made with the observer blinded with respect to the treatment.

After the first weeks of cholesterol feeding, we observed very distinct, consistent rheological changes that progressed during the course of the study. There was a dramatic decrease

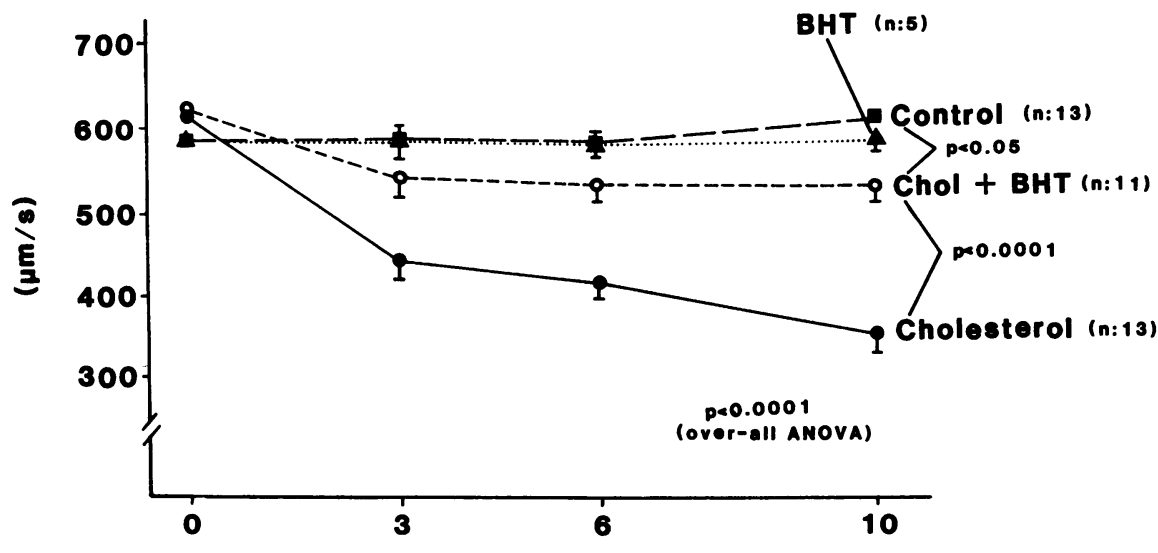

Figure 1. Effect of the diets on blood flow velocity in the conjunctival microcirculation. Blood flow velocity was determined in the conjunctival microcirculation (arterioli of the third order belonging to the anterior conjunctival artery) by the flying spot technique after different periods of time on control diet, diet containing $1 \%$ cholesterol, $1 \% \mathrm{BHT}$, and diet containing $1 \%$ cholesterol $+1 \%$ BHT. In each animal, the velocity was calculated as a mean of six independent measurements, three in each eye. Each value is the mean \pm SEM of all the animals in the group at the time given. The variation at time 0 was small enough to be contained within the symbol. For TIme (weeks) further experimental details, see Methods. 

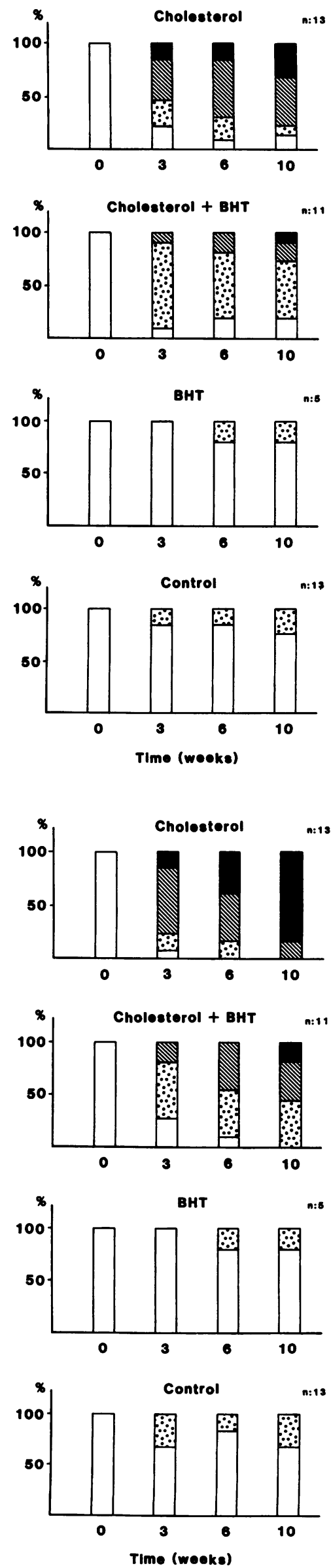

Figure 2. Occurrence of stasis in the conjunctival microcirculation in the four different groups. The occurrence of stasis in the conjunctival microcirculation was evaluated from observations on both eyes in each individual rabbit $(n=42)$ after different periods of time on the four different diets. Efforts were made to put the focus to the same area of conjunctiva on each eye of all the rabbits. The classification of the degree of stasis was as described in Methods. Group 1, white box; group 2, dotted box; group 3 , hatched box; and group 4, black box.

Figure 3. Effect of the four diets on aggregation of erythrocytes in the conjunctival microcirculation. The aggregation of erythrocytes in the conjunctival microcirculation was evaluated from observations on both eyes in each individual rabbit ( $n$ $=42$ ) after different periods of time on the four diets. Efforts were made to put the focus to the same area of conjunctiva on each eye of all the rabbits. Aggregation was classified as described in Methods. Group 1, white box; group 2, dotted box; group 3, hatched box; and group 4, black box. in blood flow velocity in the cholesterol group, accompanied by aggregation of cells in $40-50 \%$ of the small vessels (vessels smaller than arterioli of the third degree). Previously, an increased plasma viscosity as well as an increased tendency for white blood cells to adhere to the vessel walls have been described in rabbits on a high cholesterol diet (2). Also, transient thrombuslike structures formed by leukocytes were seen. Both Asano et al. (2) and Klimenko et al. (3) have reported that red blood cells seem to have an abnormal tendency to form sludges and aggregates in hypercholesterolemic rabbits. In the present study, the BHT-treated cholesterol-fed group had significantly higher blood flow and less aggregation, in spite of a lack of reduction of blood lipid levels, arguing against the contention that hyperlipidemia per se might cause these changes.

After about $3 \mathrm{wk}$ of feeding, the capillary network started to become progressively sparse, a change that occurred convincingly only in the cholesterol group. This feature could possibly be because of increased vasoconstriction on the arteriolar side or to several unfilled (and thereby invisible) capillaries due to cell aggregation, resulting in high viscosity and compromised circulation. Granulocytes and monocytes may be trapped in the microcirculation, obstruct capillaries, and thereby induce a no-reflow phenomenon (13). This might also explain the stasis observed most prominently in the cholesterol-fed rabbits.

During the course of the study, the phenomenon of segmental dilation was observed in an increasing number of vessels, mainly in the arterioles. This finding might correspond to the previously described inequality of caliber, venular sacculations, and microaneurysms in rabbits fed cholesterol-enriched chow (3). The explanation for this phenomenon remains to be established, but could possibly be because of dysfunction or damage of the endothelial cells in the vessel wall.

Interestingly, the antioxidant BHT prevented a substantial part of the above-mentioned microcirculatory changes during cholesterol feeding. The very marked effects of BHT on arteriolar diameter, blood flow velocity, and aggregation during hyperlipidemic conditions strongly suggest that a substantial part of the cholesterol-induced changes in the microcirculation is due to oxidations that can be reversed by antioxidants. High levels of cholesterol in the circulation can be expected to be associated with high levels of cholesterol and fatty acid hydroperoxides and their products, which may be cytotoxic and/or vasoactive (for review see reference 6). Alternatively, these compounds may activate lipoxygenase systems with secondary formation of other active compounds. It has been reported that there is increased lipoxygenase activity in cholesterol-rich macrophages $(14,15)$. Activation of 5-lipoxygenase, for example, by cholesterol feeding might affect the formation of leukotrienes, important modulators of the microcirculatory flow $(16,17)$. The possibility must also be considered that some of the microcirculatory changes observed upon BHT treatment of cholesterol-fed rabbits are because of perturbations of prostacyclin $\left(\mathrm{PGI}_{2}\right)$ or thromboxane $\left(\mathrm{TxA}_{2}\right)$ formation. We have previously found decreased prostacyclin production in cholesterol-fed rabbits $(18,19)$.

The decreased arteriolar diameter observed in our model could be due to a changed vascular tonus (functional change), a change in the thickness of the wall (structural change), or both. A changed vascular tonus might be a result of the defective regulation of vascular relaxation that has been reported previously under hyperlipidemic conditions $(1,4,20,21)$. It is known that the endothelium-dependent vasodilation is re- 


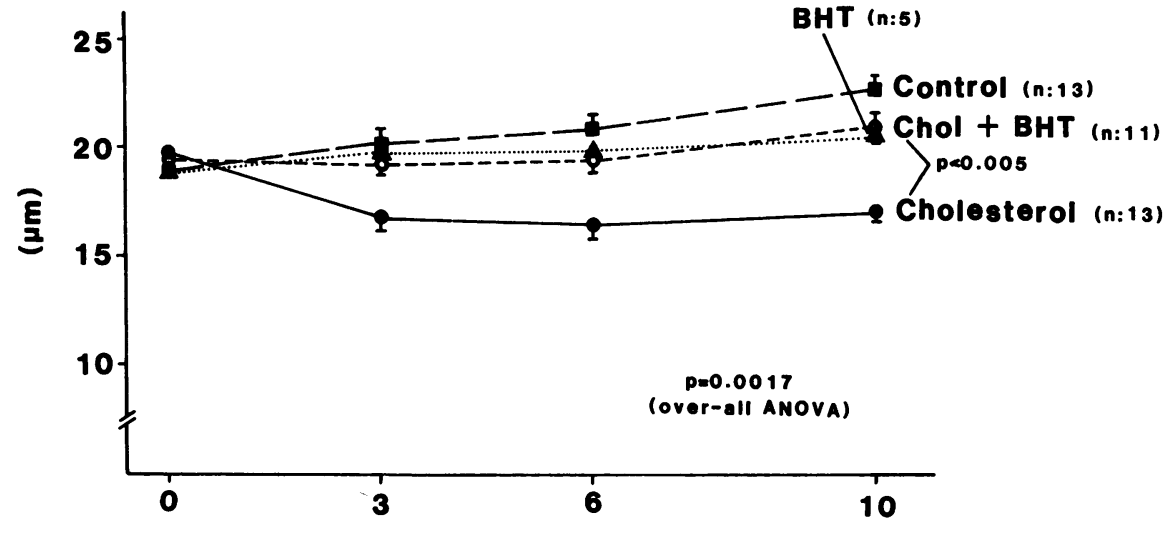

Time (weeks)
Figure 4. Effect of the diets on the average diameter of the arterial microvessels in the conjunctival microcirculation. The microvessel diameter in the conjunctival microcirculation was (arterioli of the third order belonging to the anterior conjunctival artery) measured in each individual rabbit ( $n$ $=42$ ) after different periods of time on three different diets. Each value represents the mean \pm SEM of all the animals in the group at the time given. The diameter in each animal was calculated as a mean of six independent measurements, three in each eye. An autotracking measuring technique was used as described in Methods. The $P$ value given in the figure was obtained by an overall ANOVA. duced rapidly when exposed to oxidized $\operatorname{LDL}(22,23)$. This may be because of reduced synthesis and / or increased degradation of EDRF (23). Thus, there may be a link between circulating lipid hydroperoxides and EDRF. We have found that cholesterol-treated rabbits have relatively high circulating levels of oxidized lipids as compared with rabbits treated with cholesterol/BHT (5), and higher levels of EDRF may then be expected in the latter animals.

In conclusion, we have demonstrated definite and marked changes in the microvascular system of the conjunctiva in hyperlipidemic rabbits. Such marked changes were present in all rabbits that developed atherosclerotic lesions. Antioxidants such as probucol or BHT have been demonstrated previously
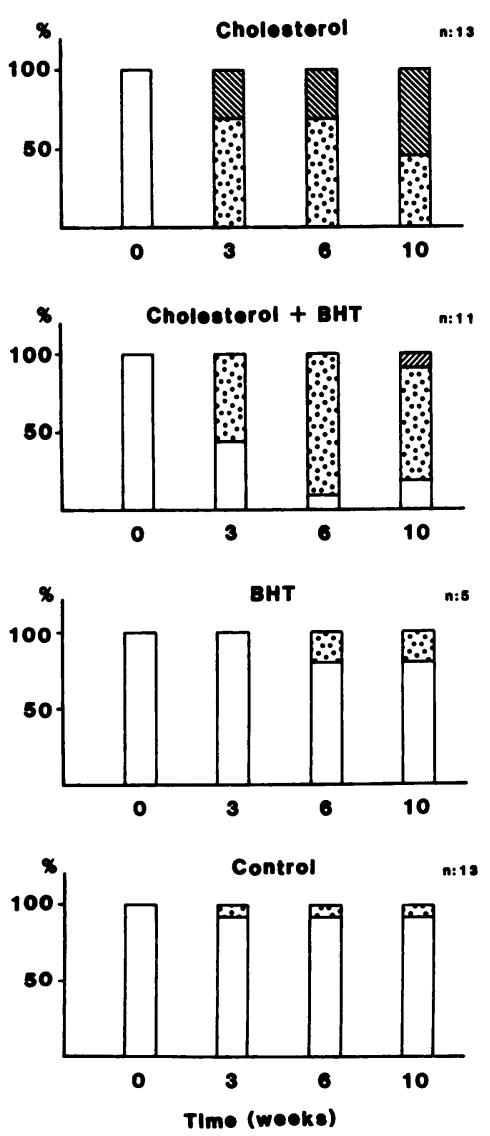

n: 13

Figure 5. Occurrence of segmental dilation in the conjunctival microcirculation in the three different groups. The segmental dilation was evaluated from observations on both eyes in the 42 rabbits, and efforts were made to put the focus to the same area of conjunctiva on each eye of all the rabbits. The degree of segmental dilation was classified as described in Methods. Group 1, white box; group 2, dotted box; group 3 , hatched box. to have antiatherogenic effects $(5,6,24)$. Although blood lipid levels remained unchanged, most of the microcirculatory changes in our study were prevented or delayed in the presence of BHT. Thus, it is tempting to suggest that oxidative mechanisms are important not only for modification of lipoproteins but also for modulation of microcirculation. Further studies are needed to clarify the possible relationship between microcirculatory changes and development of atherosclerosis.

\section{Acknowledgments}

This work was supported by the Marianne and Marcus Wallenberg Foundation, the Axelsson-Johnson Foundation, King Gustav V and Queen Viktorias Foundation, the Swedish Margarine Industry's Association for Nutritional Physiological Research, and the Swedish Medical Research Foundation.

\section{References}

1. Schuschke, D. A., I. G. Joshua, and N. F. Miller. 1991. Comparison of early microcirculatory and aortic changes in hypercholesterolemic rats. Arterioscler. Thromb. 11:154-160.

2. Asano, M., C. Ohkubo, A. Hirokawa, A. Sasaki, Y. Tochihara, and Y. Chiba. 1988. Intravital-microscopy observations on the intravascular behaviour of blood cell components during dietary-induced hyperlipidemia in the male rabbit. Biorheology. 25:329-338.

3. Klimenko, E. D., L. P. Kobozeva, A. B. Michuskaya, and I. Y. Khabarina. 1988. State of the microcirculation during long-term regression of the early stages of atherosclerosis. Byull. Eksp. Biol. Med. 105:365-368.

4. Drexler, H., A. M. Zeiher, K. Meinzer, and H. Just. 1991. Correction of endothelial dysfunction in coronary microcirculation of hypercholesterolemic patients by L-arginine. Lancet. 338:1546-1550.

5. Bjorkhem, I., A. Henriksson-Freyschuss, O. Breuer, U. Diczfalusy, L. Berglund, and P. Henriksson. 1991. The antioxidant butylated hydroxy-toluene protects against atherosclerosis. Arteriosclerosis. 11:15-22.

6. Witztum, J. L., and D. Steinberg. 1991. Role of oxidized low density lipoprotein in atherogenesis. J. Clin. Invest. 88:1785-1792.

7. Ying, X. Y., and R. J. Xiu. 1994. Dynamic and still microcirculatory image analysis for quantitative microcirculation research. In Medical Imaging 1994: Physiology and Function from Multidimensional Images. R. S. Acharaya and E. A. Hoffman, editors. Society of Photo-optical Instrument Engineers Proc. Vol. 2168. In press.

8. Ying, X., Y. Bao, R. J. Xiu, and M. Karras. 1994. Adaptive detection of microvascular edge in microcirculatory image for auto-tracking measurement of spontaneous vasomotion. In Medical Imaging 1994: Image Processing. M. H. Loew, editor. Society of Photo-optical Instrument Engineers Proc. Vol 2167. In press.

9. Magers, S., and J. E. Faber. 1992. Real-time measurement of microvascular dimensions using digital cross-correlation image processing. J. Vasc. Res. 29:241-247.

10. Mahler, F., H. Saner, C. Boss, and M. Annaaheim. 1987. Local cold 
exposure test for examination of patients with Raynaud's syndrome. Microvasc. Res. 10:422-427.

11. Gasser, P. 1990. Capillary blood flow velocity in finger nailfold: characteristics and reproducibility of the local cold response. Microvasc. Res. 40:29-35.

12. Zweifach, B. W. 1973. Microvascular aspects of tissue injury. In The Inflammatory Process. B. W. Zweifach, L. Grant, and R. T. McCluskey, editors. Academic Press Inc., New York, London. 3-46.

13. Schmid-Schönbein, G. W. 1993. The damaging potential of leukocyte activation in the microcirculation. Angiology. 44:45-46.

14. Mathur, S. N., F. J. Field, A. A. Spector, and M. L. Armstrong. 1985. Increased production of lipoxygenase products by cholesterol-rich mouse macrophages. Biochim. Biophys. Acta. 837:13-19.

15. Mathur, S. N., E. Albright, and F. J. Field. 1989. Regulation of 12-hydroxyeicosatetraenoic acid synthesis by acetyl-LDL in mouse peritoneal macrophages. Biochim. Biophys. Acta. 1001:50-59.

16. Hedqvist, P., J. Raud, and S.-E. Dahlén. 1990. Microvascular actions of eicosanoids in the hamster cheek pouch. In Advances in Prostaglandin, Throboxane and Leukotriene Research. Vol. 20. B. Samuelsson, S.-E. Dahlén, J. Fritsch, and P. Hedquist, editors. Raven Press, New York. 153-160.

17. Piper, P. J., A.-P. Sampson, H. Yaacob, and J. M. McLeod. 1990. Leukotrienes in the cardiovascular system. In Advances in Prostaglandin, Thromboxane and Leukotriene Research. Vol 20. B. Samuelsson, S.-E. Dahlén, J. Fritsch, and P. Hedqvist, editors. Raven Press, New York. 146-152.
18. Henriksson, P., M. Stamberger, and U. Diczfalusy. 1987. Increased aortic thromboxane production in experimental atherosclerosis. Prostaglandins Leukotrienes Med. 29:71-77.

19. Fogelberg, M., O. Vesterqvist, U. Diczfalusy, and P. Henriksson. 1989. Experimental atherosclerosis: effects of estrogen and atherosclerosis on thromboxane and prostacyclin production. Eur. J. Clin. Invest. 19:395-403.

20. Jayakody, L., T. M. Kappagoda, P. J. Senaratne, and A. B. R. Thomson. 1988. Impairment of endothelium-dependent relaxation: an early marker for atherosclerosis in the rabbit. Br. J. Pharmacol. 94:335-346.

21. Girerd, X. J., A. T. Hirsch, J. P. Cooke, V. J. Dzau, and M. A. Creager. 1990. L-arginine augments endothelium-dependent vasodilation in cholesterolfed rabbits. Circ. Res. 67:1301-1308.

22. Kugiyama, K., S. A. Kerns, J. D. Morrisett, R. Roberts, and P. D. Henry. 1990. Impairment of endothelium-dependent arterial relaxation by lysolecihin in modified low-density lipoproteins. Nature (Lond.). 344:160-162.

23. Chin, J. H., S. Azhar, and B. B. Hoffman. 1992. Inactivation of endothelial derived relaxing factor by oxidized lipoproteins. J. Clin. Invest. 89:10-18.

24. Carew, T. E., D. C. Schwenke, and D. Steinberg. 1987. Antiatherogenic effect of probocol unrelated to its hypercholesterolemic effect. Evidence that antioxidants in vivo can selectively inhibit low density lipoprotein degradation in macrophage-rich fatty streaks and slow the progression of atherosclerosis in the Watanabe heritable hyperlipidemic rabbit. Proc. Natl. Acad. Sci. USA. 84:77257729. 